MATHEMATICS OF COMPUTATION

Volume 68, Number 227, Pages 1303-1310

S 0025-5718(99)01071-6

Article electronically published on February 11, 1999

\title{
ON SUMS OF SEVEN CUBES
}

\author{
F. BERTAULT, O. RAMARÉ, AND P. ZIMMERMANN
}

\begin{abstract}
We show that every integer between 1290741 and $3.375 \times 10^{12}$ is a sum of 5 nonnegative cubes, from which we deduce that every integer which is a cubic residue modulo 9 and an invertible cubic residue modulo 37 is a sum of 7 nonnegative cubes.
\end{abstract}

\section{INTRODUCTION}

In 1770 E.Waring stated in "Meditationes Algebraicae" that every integer could be written as a sum of 9 nonnegative cubes. While the fact that a finite number of cubes is enough to represent any integer as a sum was proved by E.Maillet in 1895, A.Wieferich in 1909 established that 9 cubes are indeed enough (though his proof contained a gap filled by A.J.Kempner [7]). Moreover E.Landau proved in 1909 that 8 cubes are enough to represent all sufficiently large integers and L.E.Dickson showed in 1939 that the only exceptions to Landau's Theorem are the integers 23 and 239. A thorough historical account of the problem may be found in Chapter XXV of [5]. In 1941, Y.Linnik proved that every large enough integer is a sum of 7 cubes. It is conjectured that every such integer is a sum of at most 5 cubes, and heuristical (cf. [11], [2], [3]) and probabilistic (cf. [8]) arguments even suggest that integers larger that $10^{14}$ are in fact sums of 4 cubes.

In 1922 G.H.Hardy and J.E.Littlewood [6] used the circle method to get the number of distinct representations of an integer as a sum of 9 cubes, but it was only in 1986 that by sharpening this method R.C.Vaughan obtained [12] the number of representations of a large integer as a sum of 8 cubes. In 1989 the same author obtained (cf. [13] and [14]) a lower bound of the proper order of magnitude for the number of representations of a large integer as a sum of 7 cubes. Though what "large" means in the above results is not known, there is little doubt that small integers will not be covered by it.

To recall the numerical results known on sums of cubes, let us denote by $C_{k}$ a generic integer which can be written as a sum of $k$, or fewer, positive cubes. It is believed that

$$
C_{8} \leq 454, \quad C_{7} \leq 8042, \quad C_{6} \leq 1290740,
$$

and this has been verified up to $10^{7}$, cf. [9] and [11]. The first result of this work is the following theorem.

Theorem 1. Every integer between 1290741 and $3.375 \cdot 10^{12}$ is a sum of 5 cubes.

Received by the editor November 4, 1996 and, in revised form, October 28, 1997.

1991 Mathematics Subject Classification. Primary 11P05, 11Y50; Secondary 11B13, 11D25, $11 \mathrm{D} 72$.

Key words and phrases. Waring's problem for cubes, computational number theory. 
Details of the computations can be found in section V. Independent of this work, J.M.Deshouillers, F.Hennecart and B.Landreau have shown that the above theorem holds with $10^{16}$ instead of $3.375 \cdot 10^{12}$, which confirms our computations. This yields, by using the "greedy algorithm" (cf. Lemma 3), that every integer between 455 and $2.50 \cdot 10^{26}$ is a sum of 7 cubes. At the end of the paper we give a table of the integers less than $2.50 \cdot 10^{26}$ which are not sums of 7 nonnegative cubes.

The main idea involved in proving that a nonnegative integer $n$ can be written as a sum of 7 cubes relies on the identity already used by E.Maillet (cf. Lemma 6) to prove that every integer is a sum of 21 cubes. Different authors used this to deduce that every (sometimes large enough) integer in some arithmetic progression is a sum of 7 cubes. Among these the work of W.S.Baer in 1913 (cf. [1] and [7]) seems to be the most important. Y.Linnik also used a perturbation of this identity, and his proof was considerably simplified by G.L.Watson in 1951. Their proofs were ineffective. This problem was removed by K.S.McCurley in 1984; he was able to prove that every integer $\geq \exp \left(\begin{array}{lll}1 & 07734\end{array}\right)$ is a sum of 7 cubes. Even under the Generalized Riemann Hypothesis this line of attack does not offer any hope for dealing with integers $\leq 10^{100}$.

Maillet's identity leads to two problems: it requires one to know a localized divisor of an integer and to know that some cube root modulo some integer lies in a specific interval. The first problem is easily solved by noticing that any integer is a cube modulo any prime $\equiv 2[3]$ and that we know the distribution of such primes well enough. However the second one (of localized roots) remains. Note also that G.L.Watson introduced in 1951 a device which enables us to extend considerably the interval to which this root should belong. By assuming that $n$ is divisible by a small square or a small cube, we can relax this condition completely. We show here how to use another kind of argument, namely that the distribution of the cube roots of an element modulo 9 is very regular (they are well spaced). We will get

Theorem 2. Every integer which is a cubic residue modulo 9 and an invertible cubic residue modulo 37 is a sum of 7 nonnegative cubes.

The invertible cubic residues modulo 37 are $\pm 1, \pm 6, \pm 8, \pm 10, \pm 11$ and \pm 14 .

The integer 37 is not magical: it is a prime number $p_{0}$ congruent to 1 modulo 3 for which an effective version of the prime number theorem modulo $3 p_{0}$ exists. Moreover there exist three residues $r_{1}, r_{2}$ and $r_{3}$ modulo $p$ which have the same cube and any interval modulo 1 of length $\geq(3 / 4)^{\frac{1}{3}} / 2$ contains at least one of them. The limiting length is explained by Lemma 6 below. It can be shown that every large enough prime number has the last two properties, 37 and 43 being the two smallest ones, but there is no good explicit version. For the modulus $3 \times 37$ the material of [10] and some additional verifications provide us a decent explicit version.

Since every integer larger than 455 should be a sum of 7 cubes, we introduce the following parameter to quantify the portion of integers that are representable:

$$
\delta=\min _{N \geq 1} \frac{1}{N} \operatorname{Card}\{n \in[455,454+N], n \text { is a sum of } 7 \text { cubes }\},
$$

where $N$ ranges over integers. Theorem 2 immediately yields

Corollary. We have $\delta \geq \frac{4}{37}$.

Note that $\frac{4}{37}=0.108 \ldots$ 


\section{The GREEDY ALGORITHM}

Lemma 3. Assume every integer between 1290741 and $B \geq 10^{7}$ is a sum of 5 nonnegative cubes. Then every integer $n$ satisfying

$$
455 \leq n \leq\left(\frac{1}{3}(B / 3-430247)^{3 / 2}+427566\right)^{3 / 2}+8043
$$

is a sum of at most 7 nonnegative cubes.

Proof. We need a preliminary result whose proof is clear:

If $a \geq 0$ and $n \geq a+\frac{8}{27}$, then $a \leq n-m^{3} \leq a+3(n-a)^{\frac{2}{3}}$ where $m=\left[(n-a)^{\frac{1}{3}}\right]$.

Then, if $n \leq B$, there is nothing to prove. Otherwise, consider

$$
n_{1}=n-\left[(n-8043)^{\frac{1}{3}}\right]^{3} .
$$

If $n_{1} \leq B$, then the proof is over, otherwise consider further

$$
n_{2}=n_{1}-\left[\left(n_{1}-1290741\right)^{\frac{1}{3}}\right]^{3}
$$

which is $\leq B$.

For $B=3.37510^{12}$, it yields $455 \leq n \leq 2.5010^{26}$.

\section{Primes in ARIthmetic PRogressions modulo 111}

As a matter of notation, we use

$$
\theta(y ; k, \ell)=\sum_{\substack{p \equiv \ell[k] \\ p \leq y}} \log p .
$$

Lemma 4. Let $\ell$ be an integer prime to 111 . For $y \geq 247164$, the interval $\left[y, \frac{44}{43} y\right]$ contains a prime number congruent to $\ell$ modulo 111.

Proof. For $y \geq 10^{13}$, this comes from [10]:

$$
\left|\frac{\theta(y ; 111, \ell)-y}{\varphi(111)}\right| \leq 0.011261 y .
$$

For $y \in\left[247164,10^{13}\right]$, one directly constructs primes congruent to $\ell$ modulo 111 such that their successive quotients are less than $44 / 43$.

\section{REDUCTION TO A PROBLEM OF LOCALIZED DIVISORS AND LOCALIZED ROOTS}

The proof of the following lemmas draws on Watson's proof ([15]).

Lemma 5. Let $n$ be a positive integer. Assume $n \equiv 0, \pm 1[9]$. Let $t$ and $a$ be positive integers and $\beta$ be a positive real number satisfying

$$
\begin{aligned}
& \text { (i) } n-t^{3} \equiv 1[2], \quad n-t^{3} \equiv 0[3 a], \\
& \text { (ii) }(a, 6)=1, \quad\left(\frac{2}{3}\right)^{\frac{1}{3}} n^{\frac{1}{3}} \leq 3 a \leq \beta n^{\frac{1}{3}}, \\
& \text { (iii) } \frac{t}{6 a} \in\left[0, \frac{1}{2}\left(\frac{1}{\beta^{3}}-\frac{3}{4}\right)^{\frac{1}{3}}\right] .
\end{aligned}
$$

Then $n$ is a sum of seven nonnegative cubes. 
Proof. Our hypothesis on $n$ says that $n$ is a cube modulo 9. Note moreover that if $x$ is a cube modulo 9 then $x^{3} \equiv x[9]$. We thus have $0 \equiv\left(n-t^{3}\right)^{3}[9]$, i.e. $n \equiv n^{3} \equiv t^{9} \equiv t^{3}[9]$, which gives $n \equiv t^{3}[9]$. Put $N=n-t^{3}$. Then $8 N \equiv 8[16]$ and $8 N \equiv 0[9 a]$. Thus $c$ defined by $8 N=18 a\left(9 a^{2}+c\right)$ is $\equiv 3[8]$. We also have

$$
1+\frac{c}{9 a^{2}}=\frac{4}{3} \frac{n-t^{3}}{(3 a)^{3}} \in[1,2],
$$

so that $c^{2} \leq 9 a^{2}$. We can write $c^{2}=x^{2}+y^{2}+z^{2}$ with $x \equiv y \equiv z \equiv 1[2]$, and thus

$$
\begin{aligned}
n-t^{3}= & \left(\frac{3 a+x}{2}\right)^{3}+\left(\frac{3 a-x}{2}\right)^{3}+\left(\frac{3 a+y}{2}\right)^{3} \\
& +\left(\frac{3 a-y}{2}\right)^{3}+\left(\frac{3 a+z}{2}\right)^{3}+\left(\frac{3 a-z}{2}\right)^{3}
\end{aligned}
$$

as required

\section{Proof of Theorem 2 and its Corollary}

Here we assume that $n$ is a cube modulo 9 and an invertible cube modulo 37 . Let $s$ be a fixed cube root of $n$ modulo 37, the other ones being $s(-1+\sqrt{-3}) / 2$ and $s(-1-\sqrt{-3}) / 2$, where $\sqrt{-3}$ is a square root of -3 modulo 37 . Since 21 is such a square root, the three cube roots of $n$ are $s, 10 s$ and $26 s$.

We use Lemma 5 and take $a=37 \times p$, where $p$ is prime number congruent to 2 modulo 3 and congruent to the inverse of $6 s$ modulo 37 .

The system of equations $n-t^{3} \equiv 1[2]$ and $n-t^{3} \equiv 0[3 \times 37 \times p]$ admits 3 solutions modulo $2 \times 3 \times 37 \times p$, say $t_{1}, t_{2}$ and $t_{3}$. Furthermore

$$
\frac{t_{i}}{6 p \times 37} \equiv \frac{\overline{37} t_{i}}{6 p}+\frac{\overline{6 p} t_{i}}{37} \bmod 1,
$$

where $\overline{37}$ is the inverse of 37 modulo $6 p$ and $\overline{6 p}$ is the inverse of $6 p$ modulo 37 . Note first that $\frac{\overline{37} t_{i}}{6 p}$ is independent of $i$. Secondly, since $t_{i}$ modulo 37 takes the values $s, 10 s$ and $26 s$, recalling our choice of $p$, we see that the second term above is $\frac{1}{37}$, $\frac{10}{37}$ or $\frac{26}{37}$. The length of an interval modulo 1 containing none of the $\frac{t_{i}}{6 p \times 37}$ is thus $<16 / 37$, i.e. any interval having a larger length contains one of the three roots. In particular the interval $[0,16 / 37]$ contains such a root, which is the $t$ we need to apply Lemma 2 . The proof is then almost complete. Choose the parameters

$$
\begin{gathered}
\alpha=\left(\frac{2}{3}\right)^{\frac{1}{3}}, \quad \beta=\left[\left(\frac{2 \times 16}{37}\right)^{3}+\frac{3}{4}\right]^{-\frac{1}{3}} \\
\xi=\frac{\beta}{\alpha}=1.0240177 \ldots \text { and } X=\frac{(2 n / 3)^{1 / 3}}{3 \times 37} .
\end{gathered}
$$

Since $\xi>\frac{44}{43}$, Lemma 4 gives us that

$$
\theta(\xi X ; 3 \times 37, \overline{6 s})-\theta(X ; 3 \times 37, \overline{6 s})>0
$$

if $247164 \leq X$, i.e. $n \geq 3.110^{22}$, which we can assume from Theorem 1 . Lemma 2 now implies Theorem 2.

To prove its corollary, we proceed as follows. By Theorem 2, there exist at least 36 arithmetic progressions modulo 333 containing only sums of 7 cubes; moreover all integers in $[455,495]$ are sums of 7 cubes, so that $\delta_{N}=1$ for $1 \leq N \leq 41$. 
If $N$ is larger than 41 , we let $k=\left[\frac{N-41}{333}\right]$ and we have $N \delta_{N} \geq 41+36 k$ (since there are 41 sums of 7 cubes in $[455,495]$ and $k$ such sums in each of the 36 arithmetic progressions between 496 and $454+N)$. We thus have $N \delta_{N} \geq 41+36\left(\frac{N-41}{333}-1\right) \geq$ $\frac{4}{37}$.

\section{About the proof of Theorem 1}

To prove that every integer between 1290741 and $15000^{3}=3.37510^{12}$ is a sum of 5 cubes, we have first computed tables of the sums of 2,3 and 4 nonnegative cubes up to $10^{9}$ :

$\begin{array}{cccc}\text { sums of } h \text { cubes }<n & h=2 & h=3 & h=4 \\ 10^{6} & 4454 & 107875 & 713364 \\ 10^{7} & 20546 & 1037872 & 8259118 \\ 10^{8} & 95090 & 10172774 & 91057713 \\ 10^{9} & 440959 & 100735175 & 966039273\end{array}$

Then for each interval $a^{3} \leq x<(a+1)^{3}$ for $108 \leq a \leq 15000$, we have checked that $x$ can be written as a sum of five cubes, by first looking at the table of sums of four cubes for $x-a^{3}$, in case of failure for $x-(a-1)^{3}$, and so on. If the table of four cubes was too small we proceeded by subtracting the greatest cube from $x-(a-k)^{3}$, and looking in the table of sums of three cubes, and so on. Therefore we were looking for a representation $x=(a-k)^{3}+C_{4}$ - where $C_{4}$ denotes a sum of four cubes - with the smallest $k$. Let us denote this value $k(x)$.

The program that produces the tables of sums of cubes, the checking program and the histograms of $k(x)$ for $108 \leq a \leq 15000$ can be obtained from http://www.loria.fr/ ${ }^{\sim}$ zimmerma/records/cubes.html. The following table gives the value of the largest $x$ found in the interval $\left[108^{3}, 15000^{3}\right]$ such that $k(x)=m$ for each $m$ in $[6,18]$. The maximal and average values of $k(x)$ on each interval $a^{3} \leq x<(a+1)^{3}$ are displayed on Figure 1. Figure 2 shows for each $l$ in $[0-6]$ the percentage of $x$ in $\left[a^{3},(a+1)^{3}\right]$ such that $k(x)=l$.

$$
\begin{array}{crc}
m & \text { largest } & x \leq 3.37510^{12} \text { such that } k(x)=m \\
6 & \mathrm{a}=13637, & x=a^{3}+2018319=2536044484172 \\
7 & \mathrm{a}=7636, & x=a^{3}+94359676=445338035132 \\
8 & \mathrm{a}=6627, & x=a^{3}+8276314=291047090197 \\
9 & \mathrm{a}=4768, & x=a^{3}+822342=108395695174 \\
10 & \mathrm{a}=2765, & x=a^{3}+97142=21139144267 \\
11 & \mathrm{a}=2473, & x=a^{3}+784633=15124982450 \\
12 & \mathrm{a}=1921, & x=a^{3}+7033521=7095986482 \\
13 & \mathrm{a}=1185, & x=a^{3}+1002929=1665009554 \\
14 & \mathrm{a}=963, & x=a^{3}+1516531=894572878 \\
15 & \mathrm{a}=810, & x=a^{3}+1120900=532561900 \\
16 & \mathrm{a}=725, & x=a^{3}+718511=381796636 \\
17 & \mathrm{a}=645, & x=a^{3}+996097=269332222 \\
18 & \mathrm{a}=434, & x=a^{3}+225636=81972140
\end{array}
$$

It appears from Figure 2 that for $a \equiv 2[3]$, the percentage of $x$ in $\left[a^{3},(a+1)^{3}-1\right]$ such that $k(x)=2$ (i.e. $x-a^{3}$ and $x-(a-1)^{3}$ are not sums of four cubes but $x-(a-2)^{3}$ is) is much lower than for other values of $a$. 
Table of the integers which are not sum of 7 cubes and $\leq 2.5 \cdot 10^{26}$

In the second column of this table, we give the prime factor decomposition of the integer.

$$
\begin{aligned}
& 15=3 \cdot 5 \quad \equiv 6[9] \\
& 22=2 \cdot 11 \equiv 4[9] \\
& 23=23 \quad \equiv 5[9] \\
& 50=2 \cdot 5^{2} \equiv 5[9] \\
& 114=2 \cdot 3 \cdot 29 \equiv 6[9] \\
& 167=167 \equiv 5[9] \\
& 175=5^{2} 7 \quad \equiv 4[9] \\
& 186=2 \cdot 3 \cdot 31 \equiv 6[9] \\
& 212=2^{2} 53 \equiv 5[9] \\
& 231=3 \cdot 7 \cdot 11 \equiv 6[9] \\
& 238=2 \cdot 7 \cdot 17 \equiv 4[9] \\
& 239=239 \quad \equiv 5[9] \\
& 303=3 \cdot 101 \equiv 6[9] \\
& 364=2^{2} 7 \cdot 13 \equiv 4[9] \\
& 420=2^{2} 3 \cdot 5 \cdot 7 \equiv 6[9] \\
& 428=2^{2} 107 \equiv 5[9] \\
& 454=2 \cdot 227 \quad \equiv 4[9]
\end{aligned}
$$

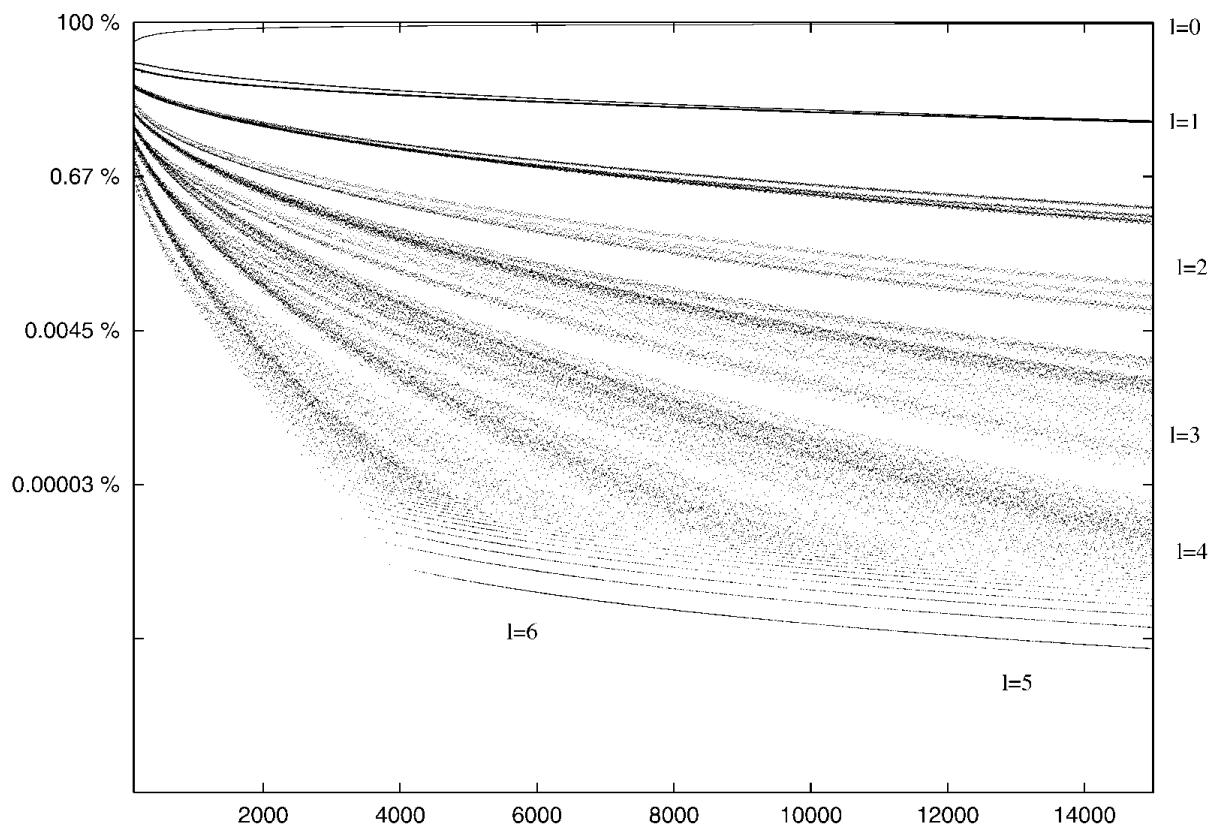

FiguRE 1. Maximal and average values of $k(x)$ on each interval $a^{3} \leq x<(a+1)^{3}$ 


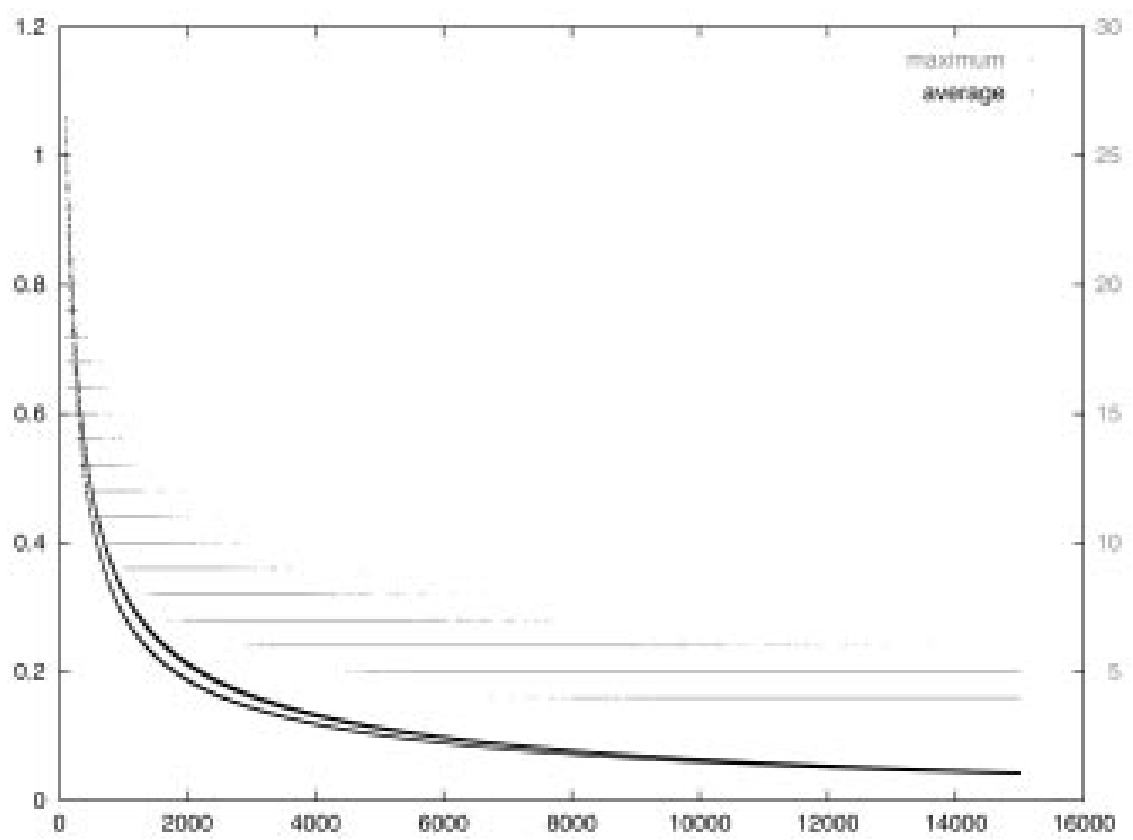

Figure 2. Percentage of $x$ in $\left[a^{3},(a+1)^{3}\right]$ such that $k(x)=l$, for each $l$ in $[0-6]$

\section{REFERENCES}

1. W.S. Baer "Über die Zerlegung der ganzen Zahlen in sieben Kuben", Math. Ann. (1913) Vol 74, pp 511-515.

2. J. Bohman and C.E. Fröberg "Numerical investigations of Waring's problem for cubes", BIT (1981) Vol 21, pp 118-122. MR 82k:10063

3. J.M. Deshouillers, F. Hennecart and B. Landreau, private communication.

4. L. E. Dickson "All integers except 23 and 239 are sums of 8 cubes", Bull. Amer. Math. Soc. (1939) Vol 45, pp 588-591. MR 1:5e

5. L. E. Dickson "Theory of numbers", Vol II, Chelsea Publishing Company (1971).

6. G. H. Hardy and J.E. Littlewood "Some problems of "Partitio Numerorum" IV. The singular series in Waring's Problem and the value of the number $G(k)$ ", Math. Zeitschrift (1922) Vol 12, pp 161-188.

7. A. J. Kempner "Über das Waringsche Problem und einige Verallgemeinerungen" Diss. Göttingen, 1912. Extract in Math. Annalen, (1912), Vol 72, pp 387.

8. B. Landreau "Modèle probabiliste pour les sommes de $s$ puissances $s$-ièmes", Compositio Math. (1995) Vol 99, pp 1-31. MR 97a:11163

9. K. S. McCurley "An effective seven cube theorem", J. Number Theory (1984) Vol 19, pp 176-183. MR 86c: 11078

10. O. Ramaré and R. Rumely "Primes in arithmetic progressions", Math. Comp. (1996) Vol 213, pp 397-425. MR 97a:11144

11. F. Romani "Computations concerning Waring's problem for cubes", Calcolo (1982) Vol 19, pp 415-431. MR 85g:11088

12. R.C.Vaughan "On Waring's problem for cubes" J. Reine Angew. Math. (1986) Vol 363, pp 122-170. MR 87j:11103

13. R. C. Vaughan "A new iterative method in Waring's problem", Acta Math. (1989) Vol 162, pp 1-71. MR 90c: 11072

14. R. C. Vaughan "On Waring's problem for cubes II" J. London Math. Soc. (1989) Vol 39, pp 205-218. MR 90c: 11073 
15. G. L. Watson "A proof of the seven cube theorem" J. London Mat. Soc. (1951) Vol 26, pp 153-156. MR 13:915a

Département de mathématiques, Université de Lille I, 59655 Villeneuve d’AscQ, FRANCE

E-mail address: Francois.Bertault@loria.fr

LORIA, BP 101, 54600 Villers-Lès-Nancy Cedex, France

E-mail address: ramare@gat.univ-lille1.fr

E-mail address: Paul.Zimmermann@loria.fr 